\title{
PREFACE
}

\section{Barriers to Cochlear Implant Access: Acknowledging the Challenges, Changing the Future}

$S_{\text {ince the earliest recipients received their }}$ devices more than 40 years ago, cochlear implantation has continued to evolve, benefitting more individuals with greater diversity of hearing loss. Originally introduced for those with bilateral profound cochlear hearing loss, cochlear implants (CIs) were considered to be the last resort when hearing aids could no longer provide any benefit. Today this perspective is considered woefully outdated. Successful recipients include those with single-sided deafness, asymmetric hearing loss, residual hearing in the implanted ear up to and including normal thresholds through $1,000 \mathrm{~Hz}$, sudden loss, long duration progressive loss, congenital loss implanted by 9 months of age, auditory neuropathy spectrum disorder (ANSD), and more. Candidacy determination is shifting from use of sentences in quiet in the best-aided condition to ear-specific word recognition scores. Electricacoustic stimulation in the same ear is possible. Notably, implantation as soon as possible once benefit is determined to be likely is encouraged and has been shown to result in better outcomes.

Yet, despite the exciting advancements that should be improving access to cochlear implantation and exponentially increasing utilization, numerous barriers persist. Misconceptions of contemporary candidacy, lack of comfort recommending $\mathrm{CI}$, inequities of health-related socioeconomic resources, outdated device labeling, restrictive insurance coverage policies, lack of clarity regarding the link to cognitive health, and lack of awareness by the public and referring providers have combined to prevent more than an estimated 1 million patients who could benefit from CI from receiving this care. ${ }^{1}$

Recognizing that knowledge is power, this issue of Seminars in Hearing explores these barriers to cochlear implantation and seeks to empower clinicians to successfully move their candidate patients past those barriers to enjoy better hearing and communication through cochlear implantation.

This edition brings together a diverse and accomplished group of audiologists, neurotologists, and public health specialists to outline the inherent problems with existing barriers, to explore the opportunities that will arise from recognizing and embracing best practices, and to deliver a case for change to increase utilization of this proven, life-changing technology.

The issue opens with an analysis and quantification of the current problem. Ashley Nassiri, MD, MBA, and colleagues map the patient experience as a way to uncover obstacles that $\mathrm{CI}$ candidates and recipients face at nearly every step of the process. Marissa Schuh, MPH, and Matthew Bush, MD, PhD, MBA, explore the disparities in cochlear implantation through the social determinants of health. Consideration is given to how these factors can influence equity in CI and how to incorporate this information in the evaluation and management of patients receiving CIs.

The focus then shifts more specifically to the adult population. Terry Zwolan, $\mathrm{PhD}$, and
${ }^{1}$ Head and Neck Institute, Cleveland Clinic, Cleveland, Ohio.

Address for correspondence: Sarah Sydlowski, Au.D., Ph.D., MBA, Cleveland Clinic, Head and Neck Institute, 9500 Euclid Avenue, Desk A-71, Cleveland, OH 44195 (e-mail: sydlows@ccf.org).
Barriers to Cochlear Implant Access: Acknowledging the Challenges, Changing the Future; Guest Editor, Sarah Sydlowski, Au.D., Ph.D., M.B.A.

Semin Hear 2021;42:309-310. C 2021. Thieme. All rights reserved. Thieme Medical Publishers, Inc., 333 Seventh Avenue, 18th Floor, New York, NY 10001, USA DOI: https://doi.org/10.1055/s-0041-1739280.

ISSN 0734-0451. 
Greg Basura, MD, discuss how the rate of improvement in adult CI outcomes has outpaced expansion of candidacy requirements of FDA labeling, Medicare, and private insurers in the United States, preventing many qualified candidates from financial coverage of CI technology. Eric Babajanian, MD; Neil Patel, MD; and Richard Gurgel, MD examine the relationship between cochlear implantation and cognition and quality of life in older adults, as well as how frailty affects outcomes for older patients with CIs. Sarah Mowry, MD, and colleagues dispel common myths and misconceptions related to the risks associated with CI surgery that may deter providers from recommending and patients from pursuing cochlear implantation. Finally, my colleagues and I describe the importance of hearing aid verification using aided speech recognition materials during hearing aid fitting and follow-up to confirm optimal functional of hearing aid benefit, both as routine practice and as an essential component of the CI candidacy evaluation.

Last but not least, challenges unique to the youngest CI candidates are discussed as Lisa Park, AuD, and colleagues highlight the limitations of FDA criteria and clinical practice for pediatric patients compared with adults as a barrier to pediatric implantation. Karen Gordon, $\mathrm{PhD}$, and colleagues make the case for the importance of access to bilateral hearing through CI in children.

We hope that by compiling in one location many of the key factors of under-utilization of CIs in the United States today, we have created an essential resource for empowering change for the industry, for our practices, and, most importantly, for the hundreds of thousands of patients who are struggling to achieve their best possible hearing and for whom cochlear implantation may be the answer.

Sarah Sydlowski, Au.D., Ph.D., M.B.A ${ }^{1}$

\section{CONFLICT OF INTEREST}

I am a consultant for Oticon Medical and Cochlear Corporation.

\section{REFERENCE}

1. Nassiri AM, Sorkin DL, Carlson ML. Current estimates of cochlear implant utilization in the United States. Otol Neurotol 2021; In press 